\title{
Satellite DNA in the Karyotype Evolution of Domestic Animals - Clinical Considerations
}

\author{
F. Adega H. Guedes-Pinto R. Chaves \\ Institute for Biotechnology and Bioengineering, Centre of Genetics and Biotechnology, \\ University of Trás-os-Montes and Alto Douro, Vila Real, Portugal
}

\section{Key Words}

Genome restructuring Karyotype evolution Satellite DNA Satellite DNA in clinical conditions

\begin{abstract}
Eukaryotic genomes contain far more DNA than needed for coding proteins. Some of these additional DNA sequences comprise non-coding repetitive DNA sequences, mostly satellite DNAs and also transposable elements usually located at the heterochromatic regions of chromosomes. Satellite DNAs consist of tandemly repeated DNA sequences inhabiting the mammalian genome, typically organized in long arrays of hundreds or thousands of copies. Different important functions have been ascribed to satellite DNA, from the imperative centromeric function in mitosis and meiosis to the recent discovery of its involvement in regulatory functions via satellite transcripts. Moreover, satellite DNAs, among other repetitive sequences, are believed to be the 'engine' triggering mammalian genome evolution. Repetitive DNAs are, most likely, the genetic factors responsible for promoting genomic plasticity and therefore higher rates of chromosome mutation. Furthermore, constitutive heterochromatin regions are thought to be 'hotspots' for structural chromosome rearrangements. A considerable collection of evidences places these sequences in the landscape of mammalian evolution. However, the mechanisms that could explain how
\end{abstract}

this alliance between chromosome evolution and satellite DNA is made are still enigmatic and subject of debate. Throughout the mammalian taxa, different patterns of chromosome evolution have been widely registered from heterochromatin additions/eliminations, Robertsonian translocations, whole-arm reciprocal translocations to tandem translocations; the fact is genome's repetitive fraction is playing a central role in mammalian genome structuring. Throughout this review we will focus on the evidences that associate satellite DNAs and constitutive heterochromatin to the process of chromosome evolution and consequently to domestic species genome's remodeling.

Satellite DNAs (satDNAs) are ubiquitous components of the eukaryotic genome, consisting of tandemly repeated DNA sequences typically organized in long monotonic arrays of hundreds or thousands of copies usually located in the heterochromatic regions of chromosomes, mainly near the centromere but sometimes at subtelomeric, or interstitial regions [Charlesworth et al., 1994; Chaves et al., 2000a; Kuhn et al., 2008]. The centromere is one of the most complex structures of the eukaryotic chromosome, and from yeast to human, this element is essential for the correct segregation of chromosomes to daughter cells in mitosis and meiosis. 
Chromosomal evolution constitutes a central point in the study of mammalian biology because of its involvement in population evolution and speciation [King, 1993]. If genomes are particularly unstable (with high rates of chromosome mutations), chromosome evolution will occur even if the conditions at the population level are more relaxed. Repetitive DNAs are, most likely, the genetic factors responsible for promoting genomic plasticity and therefore higher rates of chromosome mutation [Slamovits and Rossi, 2002]. Furthermore, constitutive heterochromatin regions are thought to be 'hotspots' for structural chromosome rearrangements [Yunis and Yasmineh, 1971; John, 1988; Chaves et al., 2004] (cf. fig. 1). This hypothesis is now supported by a crescent number of evidence, especially at the mammalian level. Different authors suggested a key role for satDNA in the origination of chromosome alterations [e.g. Wichman et al., 1991; Rossi et al., 1995] that depends, among other factors, on the ability of the satDNA to change its copy number and to mobilize through the genome [e.g. Reig et al., 1992]. It is thought that rapidly evolving satDNA families promote chromosomal rearrangements by means of their dynamic behavior among nonhomologous chromosomes and between different chromosome fields, as centromeres, arms and telomeres, these rearrangements having low effects on the euchromatic genome [Wichman et al., 1991].

In this review, we intend to summarize 'evidences' or 'hallmarks' that suggest an active role for satDNA in promoting chromosome rearrangements, involved in genome restructuring during evolution and in clinical situations. Several conjectures are now being portrayed trying to explain the dynamic role of satDNA in genome's remodeling particularly in domestic animals.

\section{The Major Genome Repetitive Component: Tandem Repeated satDNA}

Eukaryote genomes contain much more DNA than needed for coding proteins. Non-coding repetitive DNA sequences that can account for large fractions of the genome compose this 'extra' DNA [Slamovits and Rossi, 2002].

Since the first satDNA was isolated from mouse tissue, in 1966 by Waring and Britten, an interminable list of different satDNA families from species covering a large part of the mammalian domain, have been identified. One of the best known and characterized satDNAs is the primate satDNA, which is organized as 171-bp monomers and is found at the centromeres of all primate (including human) chromosomes studied so far [e.g. Manuelidis, 1976; Crovella et al., 1999].

Several unrelated families of satDNA can coexist in the same genome [Choo, 1990], thus forming a collection called a 'satellite DNA library', according to Salser et al. [1976]. A satDNA family has a definite length and sequence. The base composition of satDNAs is highly variable; they may be either rich in AT or GC sequences, or there can be observed considerable variation in mean base composition between the 2 DNA strands [Sumner, 2003].

Usually, the length of the repeating unit (monomer) ranges between hundreds to thousands of base pairs, but in some satellites, of some mammals, the length of the repeat can be as short as 6 bp [Beridze, 1986]. The total size of the tandem array varies from less than $100 \mathrm{bp}$ to over $100 \mathrm{Mb}$ [see Miklos and Gill, 1982] and the arrays can have thousands of repetitions [Slamovits and Rossi, 2002]. These very long arrays of satDNA form conspicuous blocks of differentially condensed chromatin on chromosomes.

\section{satDNA: Restructuring Genomes}

satDNA evolves according to the principles of concerted evolution, in which diverse mechanisms of nonreciprocal transfer induce a high turnover of satellite sequences [e.g. Dover, 1986; Elder and Turner, 1994].

The evolution of satellite repeats can be explained as a result of change in 2 parameters: copy number and nucleotide sequence [Ugarković and Plohl, 2002], resulting eventually in homogenization of the changes among repeats within the genome and their subsequent fixation in members of isolated reproductive populations in a process known as molecular drive [e.g. Dover, 1986; Elder and Turner, 1994]. Profiles of species-specific satDNA sequences are present in almost all taxonomic groups, and result from changes in copy number, nucleotide sequence, or composition of satellites [Ugarković and Plohl, 2002].

The ubiquitous coexistence of different satDNAs in the genomes of related species displaying different behaviors in these genomes [e.g. Meštrović et al., 1998; Nijman and Lenstra, 2001] leads to the postulation that there are no general patterns regarding the timing and extent of concerted evolution when considering different taxonomic groups [Modi et al., 2004]. These different patterns in heterochromatin and the great karyotypic variability have puzzled researchers over the time. For instance, some chromosomal conservative taxa have been shown to possess large amounts of heterochromatin [e.g. Barros and Patton, 1985], in contrast with others, where the 
amount of heterochromatin seems to have been reduced with the increase of genome restructurings.

\section{The Active Role of satDNA in the Occurrence of Chromosomal Rearrangements}

A considerable collection of evidences indicates that satDNA plays an important role in evolution of mammals by promoting chromosomal rearrangements. Direct evidences to confirm this are very difficult to ascertain. Nevertheless, it seems that chromosomal evolution can be affected by factors at genomic level (e.g. satDNAs) that will determine how frequently the changes in chromosomes will occur [Slamovits and Rossi, 2002].

Wichman et al. [1991] proposed that rapidly evolving satDNA families promote chromosomal rearrangements by means of their intragenomic movements (dynamic behavior). These rearrangements would have low effects on the euchromatic genome by keeping syntenic segments intact since the breakpoints occur in repetitive DNA blocks. In this model, the chromosome patterns would be highly variable in different lineages (fig. 2). In the other mode of genome restructuring (fig. 2) are the chromosomal conservative lineages with satDNA patterns localized only in specific chromosomal fields (i.e. pericentromeric regions). These 2 different modes of genome restructuring pinpoint the evidences found in the different mammalian groups studied so far, and thus constitute the hallmarks of genome evolution (fig. 2). If these patterns do suggest that chromosome evolution could be mediated by satDNA sequences (and/or other repeated sequences), the mechanisms that could explain how this alliance between chromosome evolution and satDNA is made are still enigmatic.

Some fields of medicine such as clinical cytogenetics including cancer cytogenetics can provide important information about the mechanisms involved in chromosome change induced by satDNA. In tumors, the high density of repetitive DNA in a given region provides 'hotspots' for homologous recombination and mediates translocation processes [Kolomietz et al., 2002]. Those and other diseases originated by chromosomal rearrangements that disrupt gene functions, cause other disturbances in gene expression, or confer to the carriers' higher risks of producing unbalanced gametes [e.g. Chen et al., 2007]. Besides, several regulatory mechanisms with consequences in development and evolution have satDNA as the main player, for example, silencing or changing of transcription patterns when genes fall within or near heterochromatic domains, as a consequence of a chromosomal rearrangement [e.g. Belyaeva et al., 1993; Slamovits and Rossi, 2002].
In the next paragraphs we will highlight the involvement of heterochromatin and satDNA in the chromosome rearrangements that shaped the genomes of domestic animals during evolution and in different clinical conditions; namely, heterochromatin additions/eliminations and translocations. Consideration goes also to the different hypotheses on the formation of translocations, as well as to the translocation breakpoints' molecular composition.

\section{Heterochromatin Additions/Eliminations}

Heterochromatin shows characteristic distribution patterns in karyotypes and contributes to the broad scattering of genome sizes [Redi et al., 2001].

Although many questions regarding satDNA and heterochromatin remain to be answered, we now know that the evolutionary history of eukaryotic genomes was largely influenced by this dynamic and multifaceted genome component, as demonstrated in a broad number of mammalian taxa.

In contrast to the autosomal conservatism, the Bovidae $\mathrm{X}$ chromosome shows a considerable diversity [e.g. Robinson et al., 1998], mostly a consequence of constitutive heterochromatin variation with respect to the amount and position (disruptions of euchromatic regions, centromere replacement, heterochromatic variation, among others) [Robinson et al., 1998; Gallagher et al., 1999; Iannuzzi et al., 2000; Chaves et al., 2004]. In 2005, Chaves et al. estab- lished that part of this constitutive heterochromatin varia- tion was due to satellite I (1.715 and 1.714 satDNA families) losses or gains during the evolution of the $\mathrm{X}$ chromosomes, which accompanied its morphological rearrangements. Furthermore, several authors [Robinson et al., 1998; Gal- lager et al., 1999; Iannuzzi et al., 2000; Chaves et al., 2004] believe that X chromosome constitutive heterochromatin has facilitated the intrachromosomal rearrangements de- tected during the evolution of this sex chromosome.

\section{Translocations}

The knowledge of the mechanisms behind a translocation formation is of crucial importance to the understanding of centromere and satellites and the investigation of chromosomal abnormalities.

There are several theories for the formation of translocations and most of them imply the involvement of repetitive DNA elements. Redi et al. [1990] suggested that these are influenced by a favorable spatial relationship of DNA sequences in the telomeric regions of 2 chromosomes in the S-phase of the cell cycle of a primitive germ 
cell, and by the characteristics of centromeric satDNA sequences of acrocentric chromosomes. Page et al. [1996] proposed that translocations form through a mechanism involving recombination of homologous sequences on different chromosomes. According to Garagna et al. [2001], the orientation and disposition of the satellite repeats in the pericentromeric regions strongly suggest the occurrence of sequence-dependent mechanisms of interchromosomal exchange between satellite blocks [Smith, 1976] promoting the Robertsonian and whole-arm recip-rocal translocations. Also the nicking activity of the CENP-B protein could play an important role in the formation of translocations involving the centromere. CENP-B protein binds the CENP-B box present within centromeric satDNA of many species [e.g. Masumoto et al., 1989; Volobouev et al., 1995] and could play an impor- tant role in recombination events leading to transloca- tions involving the centromeric region. Kipling and War- burton [1997] suggested that the dimerization ability of the CENP-B protein could not only promote misalign- ment between higherorder repeat units of satDNA on nonhomologous chromosomes, but also facilitate recom- bination, mediated by its nicking activity [Garagna et al., 2001].

For a large number of species, Robertsonian translo- cations were the engine that triggered evolution [King, 1993]. These specific rearrangements regularly involve centromeric DNA, mainly composed of sat DNA. Wheth- er this component is the cause or consequence of this re- current chromosomal rearrangement remains unclear, however.

Translocations have been observed in a large number of mammalian orders, from Rodentia [e.g. BrittonDavidian et al., 2005] to Chiroptera [Ao et al., 2006], Carnivora [e.g. Tian et al., 2004], Primates [e.g. Dutrillaux, 1979; Wien- berg, 2004 and references therein], or Cetartiodactyla [e.g. Gallagher and Womack, 1992; Chaves et al., 2004; Huang et al., 2006], among many others.

It seems that centromere-related rearrangements con- tribute to accelerated rates of chromosomal change, as evidenced by the repeated occurrence of Robertsonian translocations and whole-arm reciprocal translocations in the house mouse [Britton-Davidian et al., 2005].

\section{From an Evolutionary Point of View}

In Bovinae and Caprinae (Bovidae, Cetartiodactyla), where the number of autosomal arms has remained al- most constant at 56-58 for most species, the great varia-tions in chromosome numbers are due to centromeric fu- sions, where the $2 n=30$ chromosomes from Tragelaphus spekei (Bovidae, Tragelaphini tribe) [Wallace, 1978] can easily be deduced from the $2 \mathrm{n}=60$ chromosomes of the karyotype of Bos taurus (Bovini) or Capra hircus (Cap- rini) by a number of Robertsonian translocations [e.g. Gallagher and Womack, 1992; Chaves et al., 2004]. As examples, the karyotype of Bos javanicus birmanicus differs from that of cattle, Bos taurus, by 2 pairs of submeta- centric chromosomes [Ropiquet et al., 2008]. Also Bos gaurus shares 1 of these Robertsonian translocations with $B . j$. birmanicus [Gallagher and Womack, 1992]. In the recently characterized saola (Pseudoryx nghetinhensis), the karyotype also seems to have evolved by centro- meric fusions [Ahrens et al., 2005]. Among buffaloes (Bubalus and Syncerus genera), besides 2 tandem fusions [Di Berardino and Iannuzzi, 1981; Tanaka et al., 2000], several combinations of Robertsonian translocations from the primitive bovid karyotype occurred to give rise to the karyotype variations of the different species [Di Berardino and Iannuzzi, 1981; Gallagher and Womack, 1992; Gallagher et al., 1999; Tanaka et al., 2000]. Studies on bovine satellite IV DNA [Adega et al., 2006a] revealed the presence of this satDNA family in the genomes of water buffalo and of 2 species from the Tragelaphini tribe. The amount of this centromeric satDNA was lower in the submetacentric chromosomes of these species, pointing to its involvement in the Robertsonian evolu- tionary translocations that originated these chromo- somes. Moreover, some of them showed variations in the amount of satDNA IV, indicating different timing for the submetacentric chromosome formation, as it is believed that the amount of satDNA at the breakpoint regions decreases with time, in the process of homogenization [Chaves et al., 2000a, 2003a].

The karyotype of Ovis aries differs from that of Bos taurus or Capra hircus by 3 Robertsonian translocations involving 6 acrocentric chromosomes and within the Ovis genus, it is possible to find karyotypes presenting 1 or 2 of these submetacentric chromosomes, typical of all extant genera within the tribe Caprini [Bunch et al., 1976]. These biarmed chromosomes are likely to have arisen sequentially by fusion of monobrachial chromosome arms: the fusion to form chromosome 1 is regarded as the most ancient, chromosome 3 and then chromosome 2 fusions being more recent [Bunch et al., 1976]. Chaves et al. [2000a] reported that in sheep, a substantial 1.714 satDNA hybridization signal on biarmed chromosomes 2 
and 3 can be observed, but apparently no satellite signals at the centromere of chromosome 1 . It seems that the loss of satellite sequences is a gradual process, rather than happening exclusively at the time of the fusion (the Robertsonian translocation). Lack of satDNA on Ovis aries chromosome 1 is consistent with its older origin [Chaves et al., 2000a].

In Equidae (Perissodactyla), a similar scenario can be observed, with Equus przewalskii and E. caballus differ- ing by 1 Robertsonian translocation and numerous Rob- ertsonian translocations and tandem fusions accounting for the karyotypic differences between E. caballus and zebras (E. zebra and E. burchelli) [Yang et al., 2003].

In the Suiformes, the predominant type of evolution- ary rearrangements are tandem fusions, the number of rearrangements separating Suidae from Tayassuidae be- ing one of the highest found [Bosma et al., 2004; Adega et al., 2006b]. But also here Adega et al. [2008] investigat- ed different members of the pig (Suidae family) major satDNA families (Ac2 and Mc1) in 2 Tayassuidae ge- nomes, namely Pecari tajacu and Tayassu pecari. Surprisingly they found a dissimilar physical location of the sequences in the chromosomes of the families, display- ing a multiple distribution pattern in the peccaries' chromosomes in opposition to the centromerically located sequences in the pig. Moreover, it was possible to assign the satDNAs at the Tayassuidae/Suidae evolutionary breakpoints [Adega et al., 2007], pinpointing their impli- cation in the evolutionary rearrangements. By parsimony it is highly improbable that these sequences 'appear' at these locations by convergence, hence, they are drivers or remnants of the rearrangements.

\section{From the Clinical Point of View}

Since the first chromosome rearrangement identified in a domestic animal in the 60s [Gustavsson and Rock- born, 1964], rob(1;29) in cattle, an extensive list of trans- locations has been assembled [e.g. Ducos et al., 2008 and references therein]. Translocations and especially Robertsonian and reciprocal translocations are the rearrangements most often found in clinical conditions of livestock species. The pig (Sus scrofa) karyotype seems to be the one presenting the greatest variations, with a prevalence of $1 / 200$ of structural chromosomal rearrange- ments [Ducos et al., 2008], these being mainly reciprocal translocations, some of them involving the centromeric region [Pinton et al., 1998; Ducos et al., 2002].

In most but not all of the cases, there is a clear association of these rearrangements with several clinical conditions such as congenital malformations, intersexuality or reproductive dysfunction observed by reduction of the fertility/prolificacy of the carriers and/or of their mates [Gustavsson, 1971; Refsdal, 1976; Popescu et al.,

1984]. Heterozygotes for Robertsonian chromosomes have a tendency to be infertile or to produce offspring with birth defects. In wild mammals, Robertsonian chromosomes may contribute to reproductive isolation and thus to speciation. In humans, this has great clinical consequences; in domesticated mammals of economic importance it may lead to loss of productivity [Garagna et al., 2001].

Quite interesting is the fact that most of these supposed deleterious and de novo translocations involve the same chromosomes and apparently the same breakpoints as do the ones found as a cause of speciation in a great number of species.

Although most of these translocations involve centromeric sequences, only a small number of works devoted attention to the analysis of the breakpoint regions and to the nature of their molecular composition. Chaves et al. [2003a, b] and Di Meo et al. [2006] deeply analyzed the physical organization of satDNA distribution at centromere during Robertsonian translocations in an $8 ; 11$ translocation in Ovis aries, and in the 1;29 translocation in Bos taurus, respectively. This allowed depicting the translocation mechanisms undergone during the chromosome rearrangement and to assign the breakpoint regions.

The identified $\operatorname{rob}(8 ; 11)$ in $O$. aries showed a different organization from the biarmed chromosomes that are evolutionarily derived and provides strong evidence for an intermediate step in evolution of the biarmed chromosomes in sheep. If the ancestral satellite patterns of all acrocentric autosomes were similar, then the biarmed chromosomes 1,2 , and 3 have undergone a complex reorganization of satellite sequences during the translocation and evolution [Chaves et al., 2003b].

In the 1;29 translocation, it was possible to determine that this translocation involved at least 2 steps [Chaves et al., 2003a]. In this model, a first step explained the occurrence of the Robertsonian translocation itself (with the breakpoints that precede the rearrangement constituted of satellite III in BTA1 and satellite IV in BTA29); however, a second step (e.g. pericentric inversion) was necessary for the satDNA reorganization at the centromeric level, which highlights the active role of satDNA sequenc- 
es in the translocation and reinforces the functional meaning of this satDNAs in all these processes. In fact, the physical and organizational analyses of different satDNA families in a model translocation, like the wellknown 1;29 in cattle [Chaves et al., 2000b, 2003a; Di Meo et al., 2006], for example, can greatly increase our understanding on the translocation events during mammalian genome evolution.

\section{Conclusions}

The breakpoints of chromosomal rearrangements have been found at sites of segmental duplications [Kehrer-Sawatzki and Cooper, 2008], high concentrations of repetitive sequences [Chaves et al., 2003a, b], or between interspersed repetitive elements [Gray, 2000; Adega et al., 2007, 2008].

Recently, Ruiz-Herrera et al. [2006] analyzed the distribution of a large amount of syntenic blocks, evolutionary breakpoint regions, and evolutionary breakpoints taken from public databases available for 7 eutherian species (mouse, rat, cattle, dog, pig, cat and horse) and chick- en, and examined these for correspondence with fragile sites and tandem repeat locations in the human genome. Moreover, they found a striking correspondence between human fragile site location, the positions of evolutionary breakpoints, and the distribution of tandem repeats throughout the human genome [Ruiz-Herrera et al., 2006]. Simultaneously, the molecular characterization of chromosomal regions implicated in evolutionary breakpoints in mouse, human and primate genomes revealed that the main genome's reorganizations have a predisposition to occur at, or close to, regions rich in segmental duplications and in simple tandem repeats [Ruiz-Herrera et al., 2006 and references therein]. Furthermore, Schibler et al. [2006] have found that the breakpoint regions among cattle, human and mouse are enriched in lineage specific repeats.

These breakpoints do not seem to be uniformly distributed; on the contrary, they seem to follow a model of non-random occurrence [Kulemzina et al., 2009]. Certain human chromosomes and chromosomal regions display a high breakpoint accumulation and in some cases these seem to be conserved along chromosome evolution in different eutherian orders.

The results of Ruiz-Herrera et al. [2006] also provide evidence for the existence of chromosomal regions in the genome that have been repeatedly used, at least in humans, thus confirming and extending earlier observations of a breakpoint reuse [e.g. Murphy et al., 2005].

Although some genomes are more prone to rearrange than others, the fact is that a considerable number of chromosomal rearrangements have been described in the most important domestic species around the world. These rearrangements are not always related to infertility, disease or phenotypic abnormality, but in the great majority of cases, they are, as stated by the numerous reports, to loss of productivity.

Both from the evolutionary and the clinical point of view, it is now clear that each genome structure exhibits sensitivity to rearrangements involving certain chromosome regions, as observed in bovids where the centromere is the stage for most of the rearrangements described, or differently in pigs, where the rearrangement breakpoints seem to be located at the chromosome's arms. However, a common trait highlights in all of these cases the involvement of repetitive sequences in all these rearrangements, either centromeric or interspersed in the genome. Moreover, a large proportion of 'evidences' support the hypothesis that certain chromosomal regions are prone to breakage and reorganization not only during the evolutionary process, but also in clinical conditions, as it seems that some of these breakpoints are repeatedly used.

Molecular investigation of relevant repetitive sequences [Garagna et al., 2002; Chaves et al., 2003a, b] and comparison of evolutionary breakpoints with those that are 'disease-associated' [Kehrer-Sawatzki and Cooper, 2008] are essential to determine the existence of a 'molecular signature' for the occurrence or predisposition for these chromosomal rearrangements either in evolution or in clinical conditions and to assist in the characterization of the mechanistic similarities between them.

In this review, we tried to summarize the 'evidences' or 'hallmarks' suggesting an active role for satDNA and other repeated sequences, in promoting chromosome rearrangements and genome remodeling. In fact, it seems that the genome's repeated fraction could be the 'agent' for genome restructuring. This hypothesis is now being supported by a crescent number of evidences, especially at the mammalian level. However, further studies and the development of different methodologies are needed to as- certain which mechanisms are in fact behind this alli- ance that seems to exist between the repeated fraction and chromosomal restructuring. 


\section{Acknowledgements}

This work was partially supported by the projects POCTI/P/ BIA/11285/98 and POCI/BIA-BCM/58541/2004 of the Science and Technology Foundation (FCT) from Portugal and by a research position on Animal Genomics to the 'Sistema Científico e Tecnológico Nacional - Ciência 2007' (FCT).

\section{References}

Adega F, Chaves R, Guedes-Pinto H: Physical organization of the 1.709 satellite IV DNA family in Bovini and Tragelaphini tribes of the Bovidae: Sequence and chromosomal evolution. Cytogenet Genome Res 114:140-146 (2006a).

Adega F, Chaves R, Kof ler A, Krausman PR, Masabanda J, et al: High resolution comparative chromosome painting in the collared peccary (Pecari tajacu, Tayassuidae): a comparison with the karyotype of pig and sheep. Chromosome Res 14:243-251 (2006b).

Adega F, Chaves R, Guedes-Pinto H: Chromosomal evolution and phylogenetic analyses in Tayassu pecari and Pecari tajacu (Tayassuidae): Tales from the constitutive heterochromatin. J Genet 86:19-26 (2007).

Adega F, Chaves R, Guedes-Pinto H: Suiformes orthologous satellite DNAs as a hallmark of collared and white-lipped peccaries (Tayassuidae) evolutionary rearrangements. $\mathrm{Mi}$ cron 39:1281-1287 (2008).

Ahrens E, Graphodatskaya D, Nguyen BX, Stranzinger G: Cytogenetic comparison of saola (Pseudoryx nghetinhensis) and cattle (Bos taurus) using G- and Q-banding and FISH. Cytogenet Genome Res 111:147-151 (2005).

Ao L, Gu X, Feng Q, Wang J, O'Brien PC, et al: Karyotype relationships of six bat species (Chiroptera, Vespertilionidae) from China revealed by chromosome painting and Gbanding comparison. Cytogenet Genome Res 115:145-153 (2006).

Barros MA, Patton JL: Genome evolution in pocket gophers (genus Thomomys). III. Fluorochrome-revealed heterochromatin heterogeneity. Chromosoma 92:337-343 (1985).

Belyaeva ES, Demakova OV, Umbetova GH, Zhimulev IF: Cytogenetic and molecular aspects of position-effect variegation in Drosophila melanogaster. V. Heterochromatin- associated protein HP1 appears in euchromatic chromosomal regions that are inactivated as a result of position-effect variegation. Chromosoma 102:583-590 (1993).

Beridze T: Satellite DNA (Springer-Verlag, New York 1986).

Bosma AA, de Haan NA, Arkesteijn GJ, Yang F, Yerle M, et al: Comparative chromosome painting between the domestic pig (Sus scro$f a$ ) and two species of peccary, the collared peccary (Tayassu tajacu) and the whitelipped peccary (T. pecari): a phylogenetic perspective. Cytogenet Genome Res 105: 115-121 (2004).

Britton-Davidian J, Catalan J, Ramalhinho MG, Auffray J-C, Nunes AC, et al: Chromosomal phylogeny of Robertsonian races of the house mouse on the island of Madeira: testing between alternative mutational processes. Genet Res 86:171183 (2005)

Bunch TD, Foote WC, Spillett JJ: Translocations of acrocentric chromosomes and their impli- cations in the evolution of sheep (Ovis). Cy- togenet Cell Genet 17:122-136 (1976).

Charlesworth B, Sniegowski P, Stephan W: The evolutionary dynamics of repetitive DNA in eukaryotes. Nature 371:215-220 (1994).

Chaves R, Guedes-Pinto H, Heslop-Harrison JS, Schwarzacher T: The species and chromo- somal distribution of the centromeric - satellite I sequence from sheep in the tribe Caprini and other Bovidae. Cytogenet Cell Genet 91:62-66 (2000a).

Chaves R, Heslop-Harrison JS, Guedes-Pinto $\mathrm{H}$ : Centromeric heterochromatin in the cattle $\operatorname{rob}(1 ; 29)$ translocation: alphasatellite I se- quences, in-situ MspI digestion patterns, chromomycin staining and C-bands. Chro- mosome Res 8:621626 (2000b).

Chaves R, Adega F, Heslop-Harrison JS, Guedes- Pinto H, Wienberg J: Complex satellite DNA reshuff ling in the polymorphic $\mathrm{t}(1 ; 29)$ Rob- ertsonian translocation and evolutionarily derived chromosomes in cattle. Chromo- some Res 11:641-648 (2003a).

Chaves R, Adega F, Wienberg J, Heslop-Harrison JS, Guedes-Pinto H: Molecular cy togenetic analysis and centromeric satellite or-ganisation of a novel $8 ; 11$ translocation in sheep: A possible intermediate in biarmed chromosome evolution. Mamm Genome 14:706-710 (2003b).

Chaves R, Santos S, Guedes-Pinto H: Comparative analysis (Hippotragini versus Caprini, Bovidae) of X-chromosome's constitutive heterochromatin by in situ restriction endonuclease digestion: $\mathrm{X}$-chromosome constitutive heterochromatin evolution. Genetica 121:315-325 (2004).

Chaves R, Guedes-Pinto H, Heslop-Harrison JS: Phylogenetic relationships and the primitive $\mathrm{X}$ chromosome inferred from chromosomal and satellite DNA analysis in Bovidae. Proc Biol Sci 272:2009-2016 (2005).

Chen Y, Huang J, Liu P, Qiao J: Analysis of meiotic segregation patterns and interchromosomal effects in sperm from six males with Robertsonian translocations. J Assist Reprod Genet 24:406-411 (2007).

Choo KH: Role of acrocentric cen-pter satellite DNA in Robertsonian translocation and chromosomal non-disjunction. Mol Biol Med 7:437-449 (1990).

Crovella S, Del Pero M, Marziliano N,
Garagna S, Pecile V, et al: MFASAT: a new alphoid DNA sequence isolated from Macaca fascic- ularis (Cercopithecidae, Primates). Genome 42:1066-1070 (1999).

Di Berardino D, Iannuzzi L: Chromosome banding homologies in Swamp and Murrah buffalo. J Hered 72:183-188 (1981).

Di Meo GP, Perucatti A, Chaves R, Adega F, De Lorenzi L, et al: Cattle $\operatorname{rob}(1 ; 29)$ originating from complex chromosome rearrangements as revealed by both banding and FISH-mapping techniques. Chromosome Res 14:649655 (2006).

Dover GA: Molecular drive in multigene families: how biological novelties arise, spread and are assimilated. Trends Genet 2:159-165 (1986).

Ducos A, Pinton A, Yerle M, Séguéla A, Berland HM, et al: Cytogenetic and molecular characterization of eight new reciprocal translocations in the pig species. Estimation of their incidence in French populations. Genet Sel Evol 34:389-406 (2002).

Ducos A, Revay T, Kovacs A, Hidas A, Pinton A, et al: Cytogenetic screening of livestock populations in Europe: an overview. Cytogenet Genome Res 120:26-41 (2008).

Dutrillaux B: Chromosomal evolution in primates: tentative phylogeny from Microcebus murinus (Prosimian) to man. Hum Genet 48: 251-314 (1979).

Elder JF Jr, Turner BJ: Concerted evolution at the population level: pupfish HindIII satellite DNA sequences. Proc Natl Acad Sci USA 91: 994-998 (1994).

Gallagher DS Jr, Womack JE: Chromosome conservation in the Bovidae. J Hered 83:287-298 (1992).

Gallagher DS Jr, Davis SK, De Donato M, Burzlaff JD, Womack JE, et al: A molecular cytogenetic analysis of the tribe Bovini (Artiodactyla: Bovidae: Bovinae) with an emphasis on sex chromosome morphology and NOR distribution. Chromosome Res 7:481-492 (1999).

Garagna S, Marziliano N, Zuccotti M, Searle JB, Capanna E, Redi CA: Pericentromeric organization at the fusion point of mouse Robertsonian translocation chromosomes. Proc Natl Acad Sci USA 98:171-175 (2001).

Garagna S, Zuccotti M, Capanna E, Redi CA: High-resolution organization of mouse telomeric and pericentromeric DNA. Cytogenet Genome Res 96:125-129 (2002).

Gray YH: It takes two transposons to tango: transposable-element-mediated chromosomal rearrangements. Trends Genet 16: 461-468 (2000).

Gustavsson I: Chromosomes of repeat-breeder heifers. Hereditas 68:331-332 (1971).

Gustavsson I, Rockborn G: Chromosome abnormality in three cases of leukaemia in cattle. Nature 203:990 (1964).

Huang L, Chi J, Nie W, Wang J, Yang F: Phylogenomics of several deer species revealed by comparative chromosome painting with Chinese muntjac paints. Genetica 127:25-33 (2006). 
Iannuzzi L, Di Meo GP, Perucatti A, Incarnato D, Schibler L, et al: Comparative FISH mapping of bovid $\mathrm{X}$ chromosomes reveals homologies and divergences between the subfamilies Bovinae and Caprinae. Cytogenet Cell Genet 89:171-176(2000).

John B: The biology of heterochromatin; in Verma RS (ed): Heterochromatin: Molecular and Structural Aspects (Cambridge University Press, Cambridge 1988).

Kehrer-Sawatzki H, Cooper DN: Mosaicism in sporadic neurofibromatosis type 1: variations on a theme common to other hereditary cancer syndromes? J Med Genet 45: 622-631 (2008).

King M: Species Evolution: The Role of Chromosome Change (Cambridge University Press, Cambridge 1993).

Kipling D, Warburton PE: Centromeres, CENP- B and Tigger too. Trends Genet 13:141-145 (1997).

Kolomietz E, Meyn MS, Pandita A, Squire JA: The role of Alu repeat clusters as mediators of recurrent chromosomal aberrations in tumors. Genes Chromosomes Cancer 35:97112 (2002).

Kuhn GC, Sene FM, Moreira-Filho O, Schwarzacher T, Heslop-Harrison JS: Sequence analysis, chromosomal distribution and longrange organization show that rapid turnover of new and old pBuM satellite DNA repeats leads to different patterns of variation in seven species of the Drosophila buzzatii cluster. Chromosome Res 16:307-324 (2008).

Kulemzina AI, Trifonov VA, Perelman PL, Rubtsova NV, Volobuev V, et al: Cross-species chromosome painting in Cetartiodactyla: reconstructing the karyotype evolution in key phylogenetic lineages. Chromosome Res 17:419-436 (2009).

Manuelidis L: Repeating restriction fragments of human DNA. Nucleic Acids Res 3:30633076 (1976).

Masumoto H, Masukata H, Muro Y, Nozaki N, Okazaki T: A human centromere antigen (CENP-B) interacts with a short specific sequence in alphoid DNA, a human centromeric satellite. J Cell Biol 109:1963-1973 (1989).

Meštrović N, Plohl M, Mravinac B, Ugarkovic D: Evolution of satellite DNAs from the genus Palorus-experimental evidence for the "library' hypothesis. Mol Biol Evol 15:10621068 (1998).

Miklos GL, Gill AC: Nucleotide sequences of highly repeated DNAs; compilation and comments. Genet Res 39:1-30 (1982).

Modi WS, Ivanov S, Gallagher DS: Concerted evolution and higher-order repeat structure of the 1.709 (satellite IV) family in bovids. J Mol Evol 58:460-465 (2004).

Murphy WJ, Larkin DM, Everts-van der Wind A, Bourque G, Tesler G, et al: Dynamics of mammalian chromosome evolution inferred from multispecies comparative maps. Science 309:613-617 (2005).

Nijman IJ, Lenstra JA: Mutation and recombination in cattle satellite DNA: a feedback model for the evolution of satellite DNA repeats.
J Mol Evol 52:361-371 (2001).

Page SL, Shin JC, Han JY, Choo KH, Shaffer LG: Breakpoint diversity illustrates distinct mechanisms for Robertsonian translocation formation. Hum Mol Genet 5:1279-1288 (1996).

Pinton A, Ducos A, Séguéla A, Berland HM, Darré R, et al: Characterization of reciprocal translocations in pigs using dual-color chro- mosome painting and primed in situ DNA labelling. Chromosome Res 6:361-366(1998).

Popescu CP, Bonneau M, Tixier M, Bahri I, Boscher J: Reciprocal translocations in pigs. J Hered 75:448-452 (1984).

Redi CA, Garagna S, Capanna E: Nature's exper- iment with 'in situ' Hybridization? A hy- pothesis about the mechanism of $\mathrm{Rb}$ fusion. $\mathrm{J}$ Mol Evol 3:133-137 (1990).

Redi CA, Garagna S, Zacharias H, Zuccotti M, Capanna E: The other chromatin. Chromo- soma 110:136-147 (2001)

Refsdal AO: Low fertility in daughters of bulls with 1/29 translocation. Acta Vet Scand 17: 190-195 (1976)

Reig OA, Massarini AL, Ortells MO, Barros MA, Tiranti SI, Dyzenchauz FJ: New karyotypes and C-banding patterns of the subterranean rodents of the genus Ctenomys (Caviomor- pha, Octodontidae) from Argentina. Mam- malia 56:603-623 (1992).

Robinson TJ, Harrison WR, Ponce de Leon FA, Davis SK, Elder FF: A molecular cytogenetic analysis of $\mathrm{X}$ chromosome repatterning in the Bovidae: transpositions, inversions, and phylogenetic inference. Cytogenet Cell Ge- net 80:179-184 (1998).

Ropiquet A, Gerbault-Seureau M, Deuve JL, Gil- bert C, Pagacova E, et al: Chromosome evo-lution in the subtribe Bovina (Mammalia, Bovidae): the karyotype of the Cambodian banteng (Bos javanicus birmanicus) suggests that Robertsonian translocations are related to interspecific hybridization. Chromosome Res 16:1107-1118 (2008)

Rossi MS, Redi CA, Viale G, Massarini AI, Ca- panna E: Chromosomal distribution of the major satellite DNA of South American ro- dents of the genus Ctenomys. Cytogenet Cell Genet 69:179184 (1995).

Ruiz-Herrera A, Castresana J, Robinson TJ: Is mammalian chromosomal evolution driven by regions of genome fragility? Genome Biol 7:R115(2006).

Salser W, Bowen S, Browne D, el-Adli F, Fedoroff $\mathrm{N}$, et al: Investigation of the organization of mammalian chromosomes at the DNA se- quence level. Fed Proc 35:23-35 (1976).

Schibler L, Roig A, Mahe M-F, Laurent P, Hayes $\mathrm{H}$, et al: High-resolution comparative map- ping among man, cattle and mouse suggests a role for repeat sequences in mammalian genome evolution. BMC Genomics 7:194 (2006).

Slamovits CH, Rossi M: Satellite DNA: agent of chromosomal evolution in mammals. A re- view. J Neotrop Mammal 9:297-308 (2002). Smith GP: Evolution of repeated DNA sequences by unequal crossover. Science 191:528-535 (1976).

Sumner AT: Chromosome: Organization and Function (Blackwell Science Ltd, Oxford (2003).

Tanaka K, Yamamoto Y, Amano T, Yamagata T, Dang VB, et al: A Robertsonian translocation, $\operatorname{rob}(2 ; 28)$, found in Vietnamese cattle. Hereditas 133:19-23 (2000).

Tian Y, Nie W, Wang J, Ferguson-Smith MA, Yang F: Chromosome evolution in bears: reconstructing phylogenetic relationships by cross-species chromosome painting. Chromosome Res 12:55-63 (2004).

Ugarković D, Plohl M: Variation in satellite DNA profiles - causes and effects. ЕMBO J 21: 5955-5959 (2002).

Volobouev V, Vogt N, Viegas-Péquignot E, Malfoy B, Dutrillaux B: Characterization and chromosomal location of two repeated DNAs in three Gerbillus species. Chromosoma 104: 252-259 (1995).

Wallace C: Chromosomal evolution in the antelope tribe Tragelaphini. Genetica 48:75-80 (1978).

Waring M, Britten RJ: Nucleotide sequence repetition: a rapidly reassociating fraction of mouse DNA. Science 154:791-794 (1966).

Wichman HA, Payne CT, Ryder OA, Hamilton MJ, Maltbie M, Baker RJ: Genomic distribution of heterochromatic sequences in equids: implications to rapid chromosomal evolution. J Hered 82:369-377 (1991).

Wienberg J: The evolution of eutherian chromosomes. Curr Opin Genet Dev 14:657-666 (2004).

Yang F, Fu B, O'Brien PC, Robinson TJ, Ryder $\mathrm{OA}$, et al: Karyotypic relationships of horses and zebras: results of cross-species chromosome painting. Cytogenet Genome Res 102: 235-243 (2003).

Yunis JJ, Yasmineh WG: Heterochromatin, satellite DNA, and cell function. Structural DNA of eucaryotes may support and protect genes and aid in speciation. Science 174: 1200-1209 (1971). 


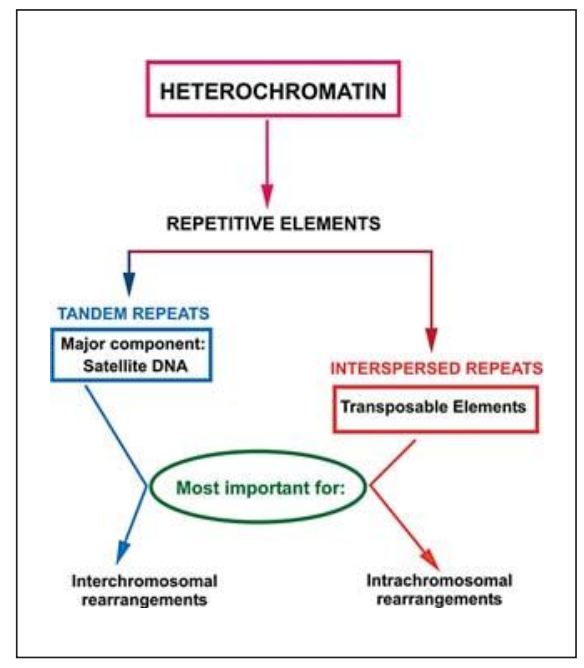

Fig. 1. Classification of the heterochromatin fraction in terms of its major sequence elements and implication in chromosome rearrangements.

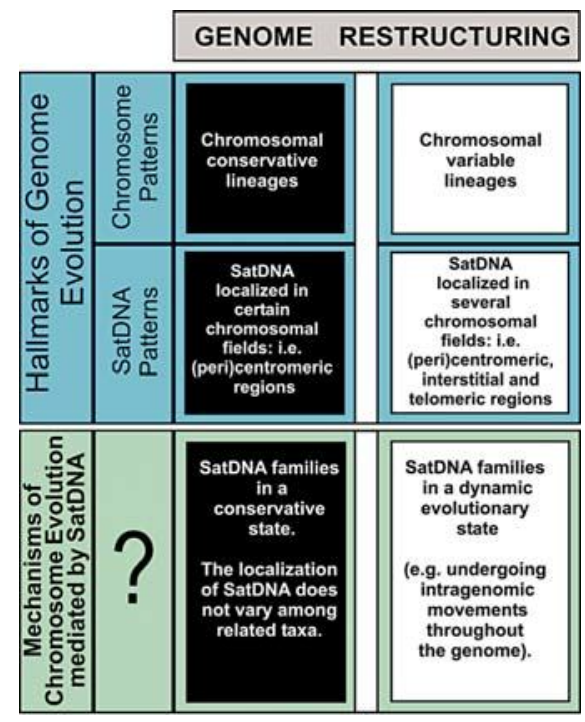

Fig. 2. The 2 main outcomes of genome restructuring. 